\title{
Rapid and Facile Purification of Apolipoprotein A-I from Human Plasma Using Thermoresponsive Nanoparticles*
}

\author{
Martin Lundqvist ${ }^{1}$, Tord Berggård ${ }^{2}$, Erik Hellstrand ${ }^{3}$, Iseult Lynch $^{1}$, Kenneth A. Dawson ${ }^{1}$, \\ Sara Linse ${ }^{3}$, Tommy Cedervall ${ }^{1,3 \#}$
}

${ }^{1}$ Centre for BioNano Interactions, School of Chemistry and Chemical Biology, University College Dublin, Dublin, Ireland; ${ }^{2}$ Department of Protein Technology, Lund University, Lund, Sweden; ${ }^{3}$ Biophysical Chemistry, Lund University Chemical Centre, Lund, Sweden. Email: "tcedervall@yahoo.com

Received February $26^{\text {th }}, 2011$; revised March $30^{\text {th }} 2011$; accepted April $18^{\text {th }}, 2011$.

\begin{abstract}
Nanoparticles can be used to purify proteins from plasma. We report here the purification of apolipoprotein A-I (apoA-I) with high specificity from human plasma using copolymeric nanoparticles. We present an optimized protocol using 50:50 NiPAM:BAM copolymer nanoparticles with thermo-responsive properties as an affinity resin. Repeated pelleting and washing of nanoparticle-captured apoA-I is achieved through temperature cycling. The protein is then eluted using urea followed by an ion exchange step for protein concentration and depletion of nanoparticles.
\end{abstract}

Keywords: Apolipoprotein A-I, Nanoparticles, Selective Purification

\section{Introduction}

The isolation and purification of proteins from complex bodily fluids is a challenging task, especially for low abundance proteins. The presence of a very high number (often several thousands) of other proteins means that classical methods like ion exchange chromatography and gel filtration yield only partial purification and a large number of steps may be needed. Affinity purification provides a shorter purification route and can lead to much simplified purification schemes if an entity with high affinity and high specificity for the desired protein is at hand. Commonly used entities are peptides, proteins, specific antibodies or low $\mathrm{Mw}$ ligands coupled to chromatography resins. For example, factor IX can be purified with vey high specificity from human plasma using conformation-specific antibodies that bind solely to the metal-stabilized conformer [1]. An example of a low $\mathrm{Mw}$ ligand is p-aminomethyl-benzene-sulfonamide for affinity purification of carbonic anhydrase [2]. Examples of

\footnotetext{
${ }^{*}$ This work was funded by an Irish Research Council for Science, Engineering and Technology Postdoctoral Fellowship (M.L.), the Marianne and Marcus Wallenberg Foundation (M.L.), the EU FP6 project NanoInteract (NMP4-CT-2006-033231), and the SFI SRC BioNanoInteract (07 SRC B1155), Centre for Nano-Vaccine, Copenhagen, Denmark, and the Swedish Research Council (VR).
}

peptides and proteins used successfully in single-step purification are the streptococcal IgA-binding peptide for human $\operatorname{IgA}$ [3], and an affibody, derived from staphylococcal protein A and selected by phage display, for Taq DNA polymerase [4]. While antibodies and other protein ligands offer very high specificity with excellent purity of the isolated protein, the scale-up costs are high because the required amount of ligand scales linearly with batch size. Development of less expensive and more versatile tools for affinity purification is therefore still a tractable goal.

Protein purification methods based on nanoparticles have recently been developed, especially nickel-ni-tri- lotriacetate modified magnetic nanoparticles for purifying poly-His-tagged proteins [5-7]. The magnetism of the particles makes the purification extremely easy. However, this approach requires engineering of the protein to be purified and is not feasible for purification of proteins from bodily fluids. Non-tagged proteins may be purified using magnetic nanoparticles modified with specific high affinity ligands as described above for chromatographic resins.

Here we report the use of thermo-responsive copolymer nanoparticles for protein purification. The special properties of NiPAM:BAM copolymer particles allow 
for repeated precipitation and re-suspension through temperature cycling, and specific interaction makes it possible to purify with high efficiency one specific protein from human plasma, apolipoprotein A-I (apoA-I). ApoA-I is the major protein constituent of high-density lipoprotein (HDL). HDL is involved in reverse cholesterol transport and is thought to play an important role in lowering the risk of coronary artery disease [8-10]. The traditional purification of apolipoproteins from plasma is a time consuming process [11-15]. The lipoproteins are normally isolated from plasma by one or several preparative ultracentrifugation steps. The purified lipoproteins contain a high degree of lipids and several proteins. The apolipoproteins are delipified using organic solvents and single apolipoproteins are separated from the mixture of proteins by biophysical and biochemical methods. This process is not protein friendly and takes days if not weeks. In contrast, the method presented here can be completed in less than 12 hours yielding apoA-I of high quality and purity.

\section{Material and Methods}

\subsection{0 nm NIPAM:BAM 50:50 Polymer Particles}

N-isopropylacrylamide-N-tert-butylacrylamide (NIPAM: BAM) copolymer particles of $70 \mathrm{~nm}$ diameter with 50:50 ratio of the monomers were synthesized in SDS micelles by free radical polymerization as described previously [16], although higher SDS concentration was used in the present work. The procedure for the synthesis was as follows: $2.8 \mathrm{~g}$ monomers (in the appropriate wt/wt ratio), and $0.28 \mathrm{~g}$ crosslinker (N,N-methylenebisacrylamide) were dissolved in $190 \mathrm{~mL}$ MilliQ water with $0.8 \mathrm{~g}$ SDS and degassed by bubbling with N2 for $30 \mathrm{~min}$. Polymerisation was induced by adding $0.095 \mathrm{~g}$ ammonium persulfate initiator in $10 \mathrm{~mL}$ degassed MilliQ water and heating at $70^{\circ} \mathrm{C}$ for 4 hours [17]. Particles were extensively dialysed against MilliQ water for several weeks, changing the water daily. Particles were lyophilized and stored in the fridge until used.

\subsection{Plasma}

Human blood was withdrawn from healthy humans into vessels pre-treated with EDTA-solution. The vessels where centrifuged for $5 \mathrm{~min}$ at 800 Relative Centrifugation Force (RCF). The supernatants (the plasma) were transferred to new vessels and stored in $-80^{\circ} \mathrm{C}$ freezer until the time of use. Before use the plasma vessel was thawed and centrifuged for $3 \mathrm{~min}$ at $16.1 \mathrm{kRCF}$ and the supernatant was transferred to a new vessel, or in the case where more than one plasma vessel was needed the supernatants were pooled.

\subsection{Particle and Plasma Mixtures}

Stock solutions of particles were made by dispersing lyophilized copolymer particles, to a concentration of 10 $\mathrm{mg} / \mathrm{mL}$, in $10 \mathrm{mM}$ Tris/ $\mathrm{HCl}, \mathrm{pH} 7.5$ with $0.15 \mathrm{mM} \mathrm{NaCl}$ and $1 \mathrm{mM}$ EDTA or in $10 \mathrm{mM}$ phosphate, $\mathrm{pH} 7.5$ with $0.15 \mathrm{mM} \mathrm{NaCl}$ and $1 \mathrm{mM}$ EDTA. The mixture was kept on ice until all the particles were dispersed (1-3 hours) before mixing with plasma. After mixing the particles and plasma the sample was kept on ice except during the centrifugation steps, which were performed at room temperature.

In one experiment, lyophilized copolymer particles were dissolved directly in plasma by adding plasma to the particles and keeping the mixtures on ice for 1 hour.

\subsection{Ion-Exchange Chromatography}

Ion exchange chromatography was performed on a HiTrap DEAE FF $1 \mathrm{~mL}$ column from GE Healthcare at room temperature in $10 \mathrm{mM}$ Tris/ $\mathrm{HCl}, \mathrm{pH} 7.5$ with $1 \mathrm{mM}$ EDTA (buffer A). Urea fractions (eluates from the nanoparticles) were pooled and passed through a $0.45 \mu \mathrm{m}$ syringe filter. After filtration the urea and salt concentrations were lowered by ten-fold dilution with buffer A. (Alternatively, the salt and urea concentration may be lowered by dialysis against buffer A). The sample was thereafter loaded onto the column. ApoA-I was eluted by stepwise increasing the $\mathrm{NaCl}$ concentration: the first step was elution with $50 \mathrm{mM} \mathrm{NaCl}$ in buffer A to remove the nanoparticles; the second step (the elution of apoA-I) is performed with $100.15 \mathrm{M} \mathrm{NaCl}$ in buffer $\mathrm{A}$.

\subsection{SDS-PAGE}

In this study two different SDS-PAGE gels have been used: PIERCE, PreciseTM Protein Gels 4\% - 20\% premade gel, which are commercially available; and $12 \%$ gels which were cast immediately prior to use.

\subsection{Commercial ApoA-I}

ApoA-I (A-0722) was obtained from Sigma-Aldrich and used as obtained.

\subsection{Biophysical Analysis by UV-, CD- and Fluorescence Spectrometry}

The commercial apoA-I (Sigma-Aldrich, A-0722) was diluted 10 times with $10 \mathrm{mM}$ phosphate, $0.15 \mathrm{M} \mathrm{NaCl}, 1$ mM EDTA, $\mathrm{pH} 7.5$, to approximately $0.15 \mathrm{mg} / \mathrm{mL}$ which corresponds approximately to the concentration of the purified apoA-I as estimated from the absorbance at 280 nm.

The CD signal between 200 and $260 \mathrm{~nm}$ was recorded on a Jasco J-720 spectropolarimeter at $25^{\circ} \mathrm{C}$ using a 1 $\mathrm{mm}$ quartz cuvette. The fluorescence emission spectra, 
after excitation at $295 \mathrm{~nm}$, were recorded between 310 and $400 \mathrm{~nm}$ on a Perkin-Elmer luminescence spectrometer LS 50B in a $1 \mathrm{~cm}$ quartz cuvette and the UV absorbance spectra were recorded between 250 and 300 in a 1 cm cuvette.

\subsection{Reconstitution of Phospholipids:ApoA-I Vesicles}

Phospholipids vesicles with apoA-I were reconstituted as previously described [18]. Briefly, apoA-I was mixed with phosphatidyl choline (PC) and minute amounts of radio-labelled 14C-POPC PC (260:1 PC/apoA-I molar ratio) and dialyzed in order to generate rHDL. The sample was separated by gel filtration on a Superose 6 column, and eluted fractions were analyzed by scintillation counting and apoA-I content was determined by apoA-I ELISA.

\section{Results and Discussion}

The purification scheme is based on the high affinity and specificity of 50:50 NiPAM:BAM copolymer nanoparticles for apoA-I $[19,20]$ and the temperature responsive behaviour of the nanoparticles. The copolymer particles are expanded and mainly dispersed as individual particles at temperatures below $10^{\circ} \mathrm{C}$, but collapsed and aggregated (at least if the particle concentration is high enough) at temperatures over $10^{\circ} \mathrm{C}$. These properties make the 50:50 NiPAM:BAM copolymer nanoparticles ideal for use in an affinity based purification scheme for apoA-I that can be completed within a day, as shown in Scheme 1.

\subsection{Purification Scheme}

The purification scheme consists of the following steps; 1) Mixing of copolymer particles with plasma and incubation on ice for 60 minutes (the mixture contains $10 \mathrm{mg}$ of 50:50 NiPAM:BAM nanoparticles of $70 \mathrm{~nm}$ diameter per $\mathrm{mL}$ human plasma); 2) Pelleting the particles by heating to room temperature and centrifugation for 3 minutes at $16 \mathrm{kRCF}$ followed by aspiration of the supernatant (which is discarded); 3) Washing of the pellet by addition of $10 \mathrm{mM}$ Tris/ $\mathrm{HCl} \mathrm{pH}$ 7.5, $1 \mathrm{mM}$ EDTA, 150 $\mathrm{mM} \mathrm{NaCl}, 100 \mu \mathrm{L}$ per mg of nanoparticles 4) Repeat step 2; 5) Repeat step 3; 6) Repeat step 2; 7) Repeat step $3 ; 8)$ Repeat step 2;9) Elution of bound protein from the nanoparticles by addition of $6 \mathrm{M}$ urea, $10 \mathrm{mM}$ Tris/HCl $\mathrm{pH} 7.5,1 \mathrm{mM}$ EDTA, $100 \mu \mathrm{L}$ per mg of nanoparticles, and incubation on ice for 30 minutes; 10) Pelleting of the particles by heating to room temperature and centrifugation for 3 minutes at $16 \mathrm{kRCF}$, followed by collection of the supernatant (saved for step 15); 11) Repeat step 9;12) Repeat step 10;13) Repeat step 9; 14) Repeat step 10;15)

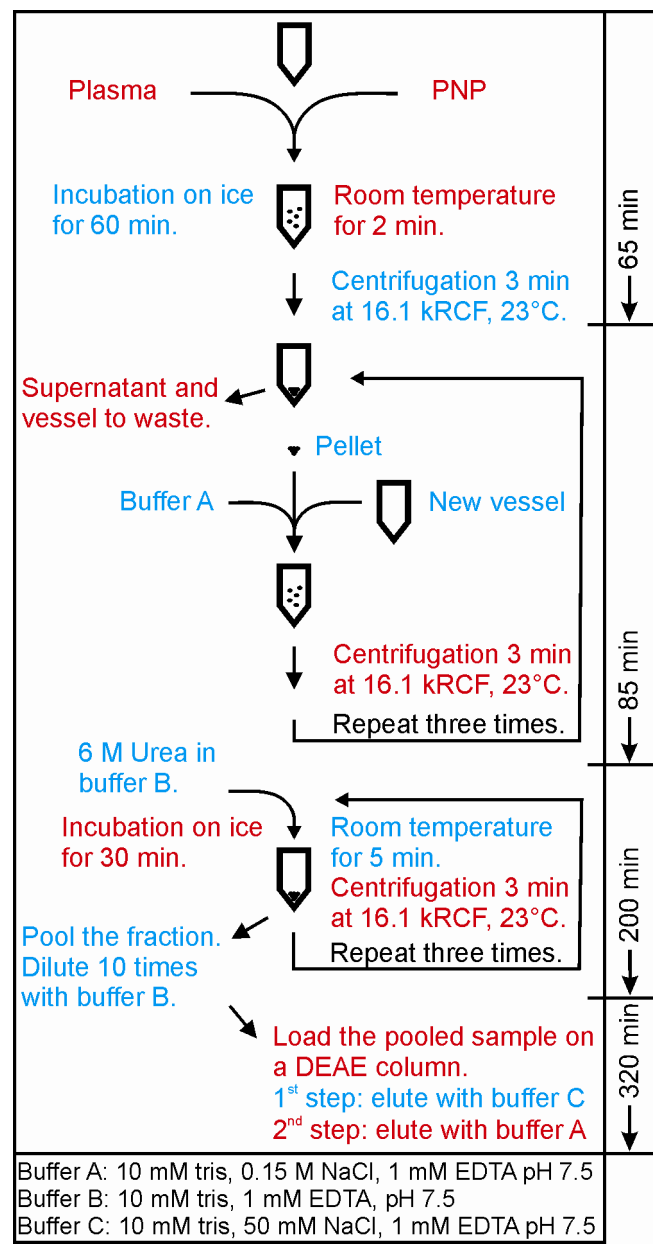

Scheme 1. The approximate experimental run time is indicated at the right-hand side of the chart.

Ten-fold dilution of the eluate (supernatant from steps 10 , 12 and 14) with $10 \mathrm{mM}$ Tris/ $\mathrm{HCl} \mathrm{pH} \mathrm{7.5,} 1 \mathrm{mM}$ EDTA, and loading onto an DEAE (HiTrap FF) ion-exchange column; 16) Washing of the column with $10 \mathrm{mM}$ Tris/HCl pH 7.5, $1 \mathrm{mM}$ EDTA, (10 mL per $1 \mathrm{~mL}$ resin); 17) 2nd washing step of the DEAE column using $10 \mathrm{mM}$ Tris/HCl pH 7.5, $1 \mathrm{mM}$ EDTA, $50 \mathrm{mM} \mathrm{NaCl},(10 \mathrm{~mL}$ per $1 \mathrm{~mL}$ resin); 18) Elution of apoA-I from the DEAE column in $10 \mathrm{mM}$ Tris/HCl $\mathrm{pH}$ 7.5, $1 \mathrm{mM}$ EDTA, 150 $\mathrm{mM} \mathrm{NaCl},(10 \mathrm{~mL}$ per $1 \mathrm{~mL}$ resin, collection of $1 \mathrm{~mL}$ fractions). Samples from all steps were analysed by SDS PAGE and the samples after steps 5, 7, 9, 11, 13, and 18 are shown in Figure 1.

The purification scheme yields pure protein free from nanoparticles, and each step has been optimized to provide the highest yield and the least disruption to the protein native state. Buffer composition, incubation time, and washing conditions were varied during this optimization and the amount of apoA-I adsorbed to the co-polymer particles was investigated using SDS PAGE. 


\subsection{Role of Salt and Buffer Composition in the Nanoparticle-Binding Step}

The results from different incubation conditions for copolymer particles with human plasma are shown in Figure 2. The effect of buffer and salt was evaluated by comparing incubation in phosphate- or trizma-based buffers at $10 \mathrm{mM}, \mathrm{pH} 7.5$ with $1 \mathrm{mM}$ EDTA, with or without $0.15 \mathrm{M} \mathrm{NaCl}$, and all solutions were incubated for 1, 3, and 6 hours. The nanoparticle bound apoA-I was eluted from the particles with SDS-loading buffer after three washing steps (using the same buffer and salt concentration as during the respective incubation). The results (Figure 2) clearly show that the buffer composition is important, as more apoA-I is retrieved from particles in Tris/ $\mathrm{HCl}$ buffers than from particles in phosphate buffer (Figure 1). The salt concentration is also impor-

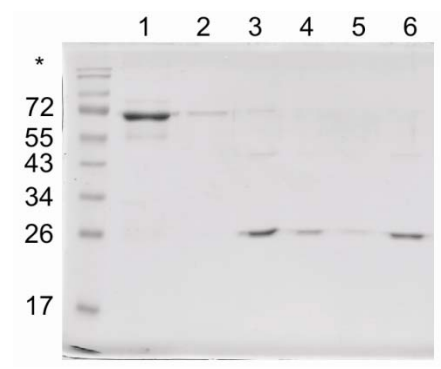

Figure 1. SDS-PAGE of samples from the different stages of the purification of apoA1. Lane 1 = supernatant after the 2nd wash (step 5), lane 2 = supernatant after the 3rd wash (step 7). Lanes 3, 4, and 5 show the supernatants after the urea elution steps $(9,11$, and 13 , respectively). Lane 6 shows the eluate of the DEAE ion-exchange column (step 17). The left lane contains molecular weight standards with the Mw of the proteins given to the left.

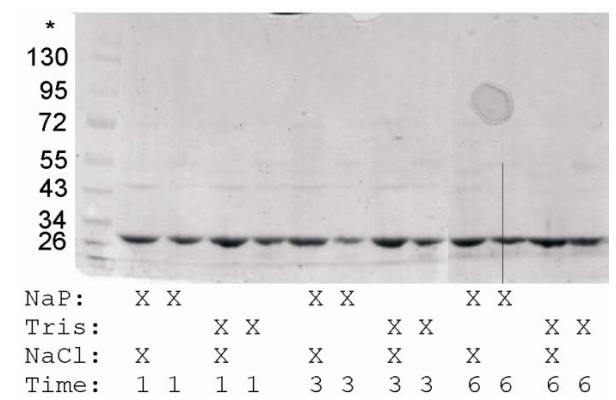

Figure 2. SDS PAGE investigation of the effects of buffer composition and incubation time. In order to study the amount of apoA-I bound to the nanoparticles proteins were eluted from the nanoparticles into SDS loading buffer after three washes using the same buffer as in the incubation step. $\mathrm{NaP}=10 \mathrm{mM}$ sodium phosphate buffer, pH 7.5, $1 \mathrm{mM}$ EDTA; Tris $=10 \mathrm{mM}$ trizma base $/ \mathrm{HCl}, \mathrm{pH} 7.5,1 \mathrm{mM}$ EDTA; $\mathrm{NaCl}=0.15 \mathrm{M} \mathrm{NaCl}$; Time $=$ incubation time in hours. The left lane contains molecular weight standards with the Mw of the proteins given to the left. tant, and the best buffer condition for the initial incubation step is $10 \mathrm{mM}$ Tris $/ \mathrm{HCl}, 1 \mathrm{mM}$ EDTA, $0.15 \mathrm{M}$ $\mathrm{NaCl}$. We also find that one hour incubation is sufficient to maximize the amount of apoA-I bound to the copolymer particles. Longer incubation does not increase the amount of apoA-I retrieved.

After incubation, pelleting and aspiration of the supernatant, apoA-I is the dominant protein bound to the nanoparticles, but serum albumin is often a contaminant. Cedervall et al. showed [20] that serum albumin dissociates much faster from the copolymer particle surface than apoA-I. This difference allows for serum albumin to be washed away while the major fraction of apoA-I remains attached to the copolymer particles.

\subsection{Role of Salt and Buffer Composition in the Nanoparticle-Washing Step}

To optimize the washing procedures, buffers with different $\mathrm{NaCl}$ concentrations, 0.15, 0.5, 1.0, and 2.0 M, were tested. As shown in Figure 3 there are no dramatic differences after the first wash, while after the second wash 1 and $2 \mathrm{M} \mathrm{NaCl}$ leads to more efficient removal of serum albumin than 0.15 and $0.5 \mathrm{M} \mathrm{NaCl}$. In the third wash, there is too little protein to allow for any quantitative comparison, and there is no obvious difference in the amount of apoA-I retrieved from the nanoparticles. Figure 3 also shows that the amount of apoA-I that is lost in the three wash steps is virtually the same for all four $\mathrm{NaCl}$-concentrations. For simplicity $10 \mathrm{mM}$ Tris/ $\mathrm{HCl} \mathrm{pH}$ 7.5, $1 \mathrm{mM}$ EDTA, $0.15 \mathrm{M} \mathrm{NaCl}$ was selected for both the sample incubation and washing steps. This buffer condition is also favorable for the subsequent purification steps (see below).

\subsection{Optimization of the Elution Step}

In order to optimize the elution of apoA-I from the

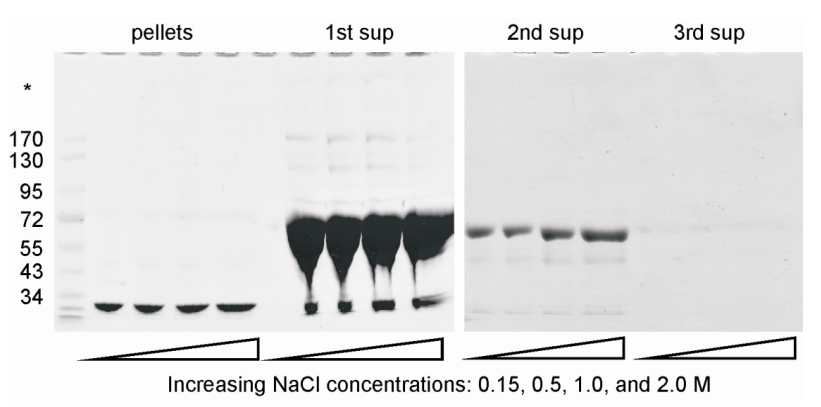

Figure 3. Optimization of the $\mathrm{NaCl}$ concentration during the washing steps. SDS PAGE of the protein eluted from the nanoparticle pellet after three washes (lanes 2-5), and of the supernatant from the 1st (lanes 7-10), 2nd (lanes 11-14) and 3rd (lanes 15-18) washing steps. The $\mathrm{NaCl}$ concentration was $0.15,0.5,1.0$ or $2.0 \mathrm{M}$ from left to right among the four samples at each step. 
nanoparticles, at least two factors are necessary to consider in order to maximize the protein yield. The efficiency of the elution is, of course, important for the final protein yield. However, the buffer composition after the elution is also important for the protein yield as it will affect the subsequent purification steps. Buffers with high salt concentration, high and low $\mathrm{pH}$, different detergents, or urea were tested for their elution efficiency (Figure $\mathbf{S 3}$ in supplementary material), determined as the highest amount of protein in the gel bands. The highest amount of apoA-I was eluted using detergents, followed by $3 \mathrm{M} \mathrm{NaCl}$, followed by $6 \mathrm{M}$ urea. At lower $\mathrm{NaCl}$ concentration, or at $\mathrm{pH} 4$ or 9.2 , little or no elution could be observed. Urea was chosen as eluant for the purification scheme as it allows the eluted proteins to be loaded directly onto an ion exchange column without changing the buffer. The detergents, although they are the best eluants, were not chosen as it could be difficult to remove them from the protein, resulting in a lower purity of the final product.

Figure 1 shows the different stages in the purification of apoA-I schematically. The amount of eluted apoA-I decreases in each step of elution with $6 \mathrm{M}$ urea (Figure 1, Lanes 3-5). The small amount of apoA-I remaining in the third urea eluate does not motivate a fourth urea step.

\subsection{Ion Exchange Chromatography}

After elution of apoA-I from the copolymer particles, ion-exchange (DEAE) chromatography is used to separate apoA-I from impurities, mainly the proteins apolipoprotein E and serum albumin [19] and copolymer particle fragments and to remove urea and change the buffer. Before loading onto the DEAE-column the pooled sample is diluted 10 times with $10 \mathrm{mM}$ phosphate buffer $\mathrm{pH}$ 7.5, $1 \mathrm{mM}$ EDTA, to reduce the $\mathrm{NaCl}$ concentration. Bound molecules are eluted from the DEAE-column in a stepwise manner. Non-protein substances are eluted in buffer with $50 \mathrm{mM} \mathrm{NaCl}$, apoA-I is eluted in buffer with $0.15 \mathrm{M} \mathrm{NaCl}$, and serum albumin elutes in $3 \mathrm{M} \mathrm{NaCl}$, which regenerates the column (serum albumin would elute much earlier if a gradient elution profile were used).

If the first step (elution in $50 \mathrm{mM} \mathrm{NaCl}$ ) is not included, something that scatters light under $270 \mathrm{~nm}$ is coeluted with apoA-I (data not shown). The fraction from the first elution step does not contain protein (no visible bands in a coomassie stained gel). Analysis by NMR spectroscopy (data not shown) indicates an interaction between buffer molecules and very large molecules and/ or a slight increase in the solution viscosity evidenced as a slower tumbling of the molecules and a broader peak [21]. The most probable explanation for the observed phenomena is that the eluted fraction consists of fragments of the copolymer particles. However, this has to be investigated further.

\subsection{Maximizing ApoA-I Yield}

Figure 3 shows that even though apoA-I has a high affinity for the copolymer particle surface, a large fraction of the apoA-I is washed away in the first washing step. Two experiments were conducted to maximize the yield of purified apoA-I. The effect of the copolymer particle concentration was checked by keeping the total sample volume and the total plasma concentration constant while the concentration of particles was varied. After incubation, the same amount (by weight) of copolymer particles was removed from each sample and the amount of bound apoA-I analyzed by SDS PAGE after elution in SDS loading buffer. The results show that the total binding of apoA-I increases with the particle concentration (Figure S1, supplementary material), hence, one limiting factor in the yield is the available particle surface area. To confirm this result a second experiment was conducted in which different amounts of lyophilized copolymer particles were dispersed in the same volume of plasma. The trend of increasing yield of apoA-I with increasing amount of available surface area was confirmed (Figure S2, supplementary material). Table 1 shows the amounts of purified apoA-I for two experiments with different particle concentrations. The purification efficiency, in percent, is calculated assuming that the concentration of apoA-I in the original plasma is $\sim 1 \mathrm{mg} / \mathrm{ml}$. These results show that it is not the apoA-I concentration in blood that is the limiting factor for the yield of purified apoA-I, but the solubility (dispersability) of the copolymer particles

Table 1. Comparison of the efficiency of the copolymer nanoparticles at purifying apoA-1 from plasma with two different dispersion methods-with TBS as an intermediate step, or direct dispersion in plasma.

\begin{tabular}{|c|c|c|}
\hline & $1^{\mathrm{a}}$ & $2^{\mathrm{b}}$ \\
\hline Theoretical max apoA-I $(\mathrm{mg})^{\mathrm{c}}$ & 3.6 & 2.2 \\
\hline Particles $(\mathrm{mg})^{\mathrm{d}}$ & 45 & 22 \\
\hline Plasma/particles $(\mathrm{ml} / \mathrm{mg})^{\mathrm{e}}$ & 0.08 & 0.1 \\
\hline Amount purified apoA-I $(\mu \mathrm{g})^{\mathrm{f}}$ & 224 & 277 \\
\hline Purification efficiency $(\%)^{g}$ & 6 & 13 \\
\hline Purified apoA-I/amount particles $(\mu \mathrm{g} / \mathrm{mg})^{\mathrm{h}}$ & 5 & 13 \\
\hline
\end{tabular}

${ }^{\mathrm{a} C}$ Copolymer particles first dispersed in TBS before mixing with plasma; ${ }^{\mathrm{b}}$ Copolymer particles directly dispersed in plasma; ${ }^{\mathrm{C}} \mathrm{Calculated}$ at a nominal apoA-I concentration of $1 \mathrm{mg} / \mathrm{ml}$ plasma; ${ }^{d}$ Total amount of copolymer particles used in the experiment; ${ }^{\mathrm{e}}$ Ratio between plasma and copolymer particles in the experiment; ${ }^{\mathrm{f}}$ Total amount of purified apoA-I in the experiment; ${ }^{g}$ Purification efficiency in percent purified apoA-I compared to the nominal apoA-I concentration; ${ }^{\mathrm{h}}$ Amount purified apoA-I per mg copolymer particles. 


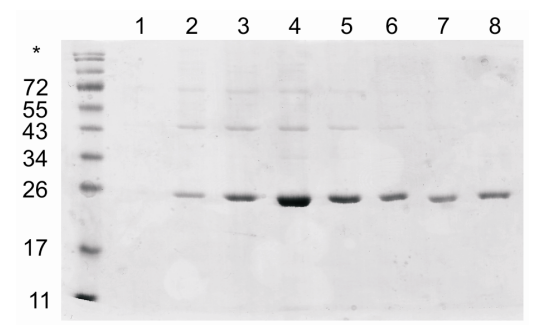

Figure S1. ApoA1 retrieved with different amounts of polymer particles added to $210 \mu \mathrm{l}$ plasma in a total volume of $910 \mu \mathrm{l}$. Lanes $1,2,3,4,5,6,7$, and 8 represent $0.007,0.07$, $0.14,0.35,0.7,1.4,2.1$, and $7.0 \mathrm{mg}$ co-polymer particles, respectively. The $0.35,0.7,1.4,2.1$ and $7.0 \mathrm{mg}$ samples contain protein from an identical amount of polymer particles.

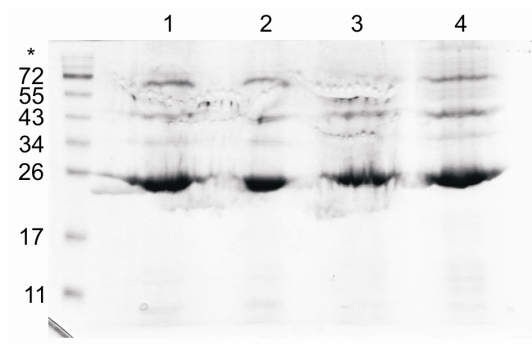

Figure S2. Lanes 1, 2, 3, and 4 represent 10, 5, 2.5 and $1 \mathrm{mg}$ co-polymer nanoparticles, respectively, that were dissolved in $1 \mathrm{ml}$ plasma. Washed with $3 \times 1000,500,250$ and $100 \mu \mathrm{L}$, respectively, of $10 \mathrm{mM}$ trizma/HCl $\mathrm{pH} 7.5,1 \mathrm{mM}$ EDTA, $0.15 \mathrm{M} \mathrm{NaCl}$. The pellets were dissolved in 100, 50, 25 and $10 \mu \mathrm{L}$ SDS-loading buffer, respectively, and $10 \mu \mathrm{L}$ of each sample was loaded on the electrophoresis gel.

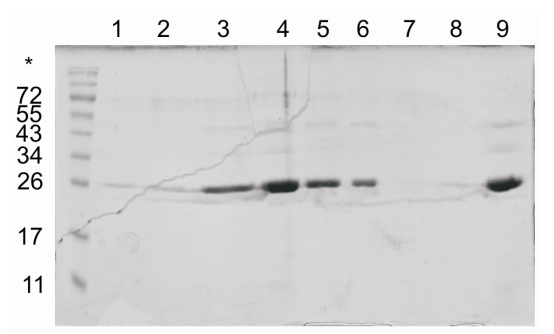

Figure S3. Elution of apoA1 from the co-polymer particles using different agents. Lanes 1, 2, and 3 are eluates in 1, 2, and $3 \mathrm{M} \mathrm{NaCl}$, respectively, lane 4 the eluate with $2 \%$ triton, lane 5 with $2 \%$ tween-20, lane 6 with $6 \mathrm{M}$ urea, lane 710 mM sodium acetate buffer, pH 4, lane $810 \mathrm{mM}$ Tris/HCl pH 9.2, and lane 9 buffer with $2 \%$ SDS. All eluates except in lanes 7 and 8 contain $10 \mathrm{mM}$ Tris/HCl pH 7.5.

in plasma and thus the amount of available surface area. The highest concentration of particles that can be dispersed in plasma is $\sim 10 \mathrm{mg}$ per $\mathrm{ml}$, as above at this concentration particle aggregation occurs, and thus the overall surface area available for bind decreases again.

\subsection{Lipid-Free ApoA-I}

Preliminary results indicate that the particles bind apoA-I together with lipids in the first step of the purification scheme. As the goal was to purify lipid free apoA-I, the final purified sample was checked for cholesterol and triglycerides with commercial enzyme-based detection kits (see SI). No lipids were detected in the purified apoA-I samples suggesting that the purified apoA-I is lipid free.

\subsection{Comparison with Commercial ApoA-I Integrity, Structure and Function}

The purified apoA-I was compared to commercially available apoA-I. On a coomassie stained 1D SDS-PAGE gel, the commercial protein gives rise to three bands, while the protein purified by the method described here gives rise to one band on the same gel with similar amounts of sample loaded, as shown in Figure 4(a). The identity of the purified apoA-I has been confirmed repeatedly by mass spectrometry $[21,22]$. The apparent differences in size between the three bands in the commercial sample of apolipoprotein A-1 may be due to modifications or proteolysis. Another explanation may be that the copolymer particles specifically target a subpopulation of apoA-I, for example, the fraction with oxidized side chains. This is less likely as the apoA-I found in the wash fractions has the same mobility as the purified protein. Thus, the three bands in commercial apoA-I are most likely due to the harsh conditions used during the existing purification process, whereas the protocol presented here leads to a homogeneous protein preparation.

Three different spectroscopic methods, UV absorbance, intrinsic tryptophan fluorescence and far UV circular dichroism spectroscopy, are used to evaluate and compare the structure of apoA-I purified via the method presented here with commercial apoA-I. The UV absorbance spectra, Figure 4(b), gives information on local structural changes around aromatic amino acids; the intrinsic tryptophan fluorescence spectra, Figure 4(c), gives information on changes in the tertiary structure; and the far UV circular dichroism spectra, Figure 4(d), reports on changes in the secondary structure. In all of the spectra there are no or very small deviations between the commercial and purified apoA-I, indicating that the tertiary and secondary structure of apoA-I are essentially the same in the two samples.

The phospholipids binding function of the purified apoA-I was examined by reconstitution of phospholipids:apoA-I vesicles. Figure $\mathbf{S} 4$ shows that the copolymer particle purified apoA-I interacts with and forms vesicles together with phospholipids in a similar way to that shown previously for apoA-I purified with a traditional purification protocol [18]. 


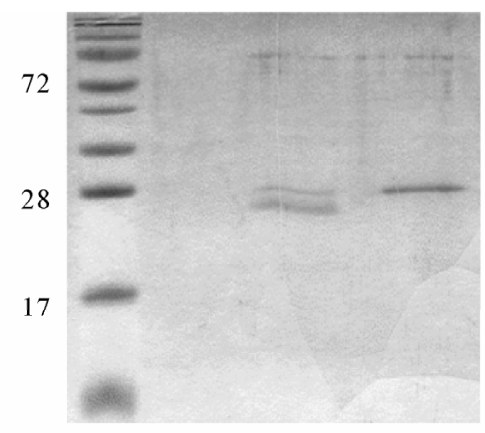

(a)

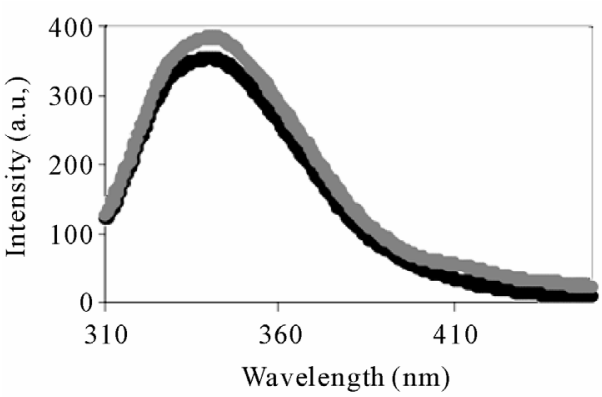

(c)

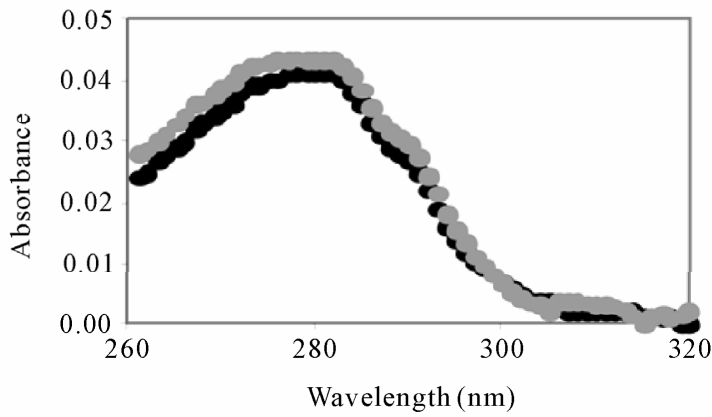

(b)

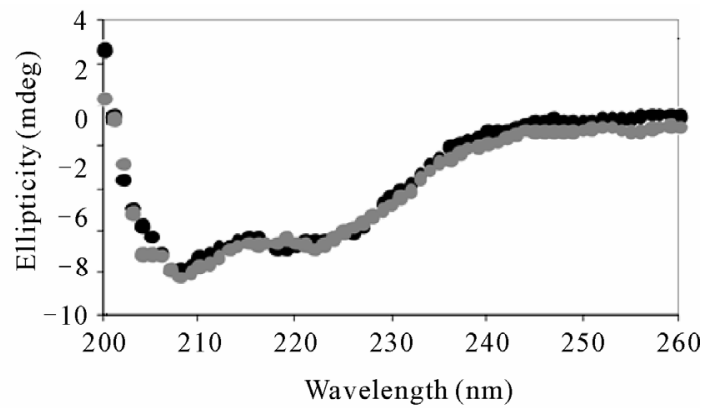

(d)

Figure 4. Comparison between commercial apoA-I and the apoA-I purified by the method described here. (a) Lane $1=$ molecular weight standards with the $\mathrm{Mw}$ of the proteins given to the left, lane $2=$ commercial apoA-I, lane $3=$ apoA-I purified in this work. In (b), (c) and (d), dark grey $=$ commercial apoA-I and light grey $=$ purified apoA-I. (b) absorbance, (c) fluorescence and $(d)$ : circular dichroism spectra.

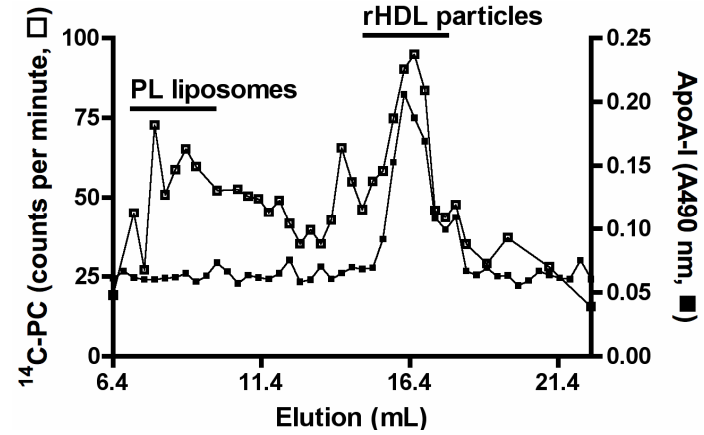

Figure S4. ApoA1 was mixed with labelled PL in order to generate rHDL. The sample was analyzed by gelfiltration. Eluted fractions were analyzed by scintillation counting and apoA1 ELISA.

\section{Conclusions}

Copolymer nanoparticles offer efficient and facile purification of apoA-I from complex protein mixtures, such as plasma. The purification scheme presented here dramatically reduces the time needed for purification. The final yield of pure apoA-I is up to $13 \mathrm{mg} / \mathrm{g}$ nanoparticles, which is comparable to other affinity chromatography systems. The particles also bind apolipoprotein E, A-IV, and A-II with high specificity. These apolipoproteins are in much lower concentrations in plasma and are therefore a challenge to purify. However, in a scaled up purification protocol it should be possible to purify also these proteins using the copolymer particles. Additionally, slight modification of the comonomer ratio could be used to optimize the particles for selective binding of these proteins.

The copolymer particles are easy and inexpensive to produce and their physical and chemical characteristics (including surface charge and/or hydrophobicity or surface energy) are easy to vary. Additionally, the particles can be modified with ligands with high specificity for selected target proteins. These features make the copolymer particles an attractive choice for purification of proteins, not limited to apolipoproteins.

\section{Acknowledgments}

We thank Professor Björn Dahlbäck and Dr Cecilia Oslakovic for help with the reconstitution of phospholipid:apoA-I vesicles and for valuable comments on the manuscript. Access to and use of UCD Conway Mass Spectrometry Resource instrumentation is gratefully acknowledged. 


\section{Supporting Information Available}

Supplementary information regarding the optimization of the purification protocol is presented. Figures showing the effects of particle concentration on apoA-I purity (Figure S1), and yield (Figure S2) and the effectiveness of various agents to elute the purified apoA-I from the particles (Figure S3), and the reconstituted phospholipids:apoA-I vesicles (Figure S4).

\section{REFERENCES}

[1] H. A. Liebman, S. A. Limentani, B. C. Furie and B. Furie, "Immunoaffinity Purification of Factor-Ix (Christmas Factor) by Using Conformation-Specific Antibodies Directed against the Factor-Ix - Metal-Complex," Proceedings of the National Academy of Sciences of the United States of America, Vol. 82, No. 11, 1985, pp. 3879-3883. doi:10.1073/pnas.82.11.3879

[2] S. O. Falkbring, P. O. Göthe, P. O. Nyman, L. Sundberg and J. Porath, "Affinity Chromatography of Carbonic Anhydrase," FEBS Letters, Vol. 24, No. 2, 1972, pp. 229235. doi:10.1016/0014-5793(72)80773-7

[3] C. Sandin, S. Linse, T. Areschoug, J. M. Woof, J. Reinholdt and G. Lindahl, "Isolation and Detection of Human IgA Using a Streptococcal IgA-binding Peptide," Journal of Immunology, Vol. 169, No. 3, 2002, pp. 1357-1364.

[4] K. Nord, E. Gunneriusson, J. Ringdahl, S. Ståhl, M. Uhlen and P.-A. Nygren, "Binding Proteins Selected from Combinatorial Libraries of an [alpha]-Helical Bacterial Receptor Domain," Nature Biotechnology, Vol. 15, No. 8, 1997, pp. 772-777. doi:10.1038/nbt0897-772

[5] H. W. Gu, K. M. Xu, C. J. Xu and B. Xu, "Biofunctional Magnetic Nanoparticles for Protein Separation and Pathogen Detection," Chemical Communications, Vol. 9, 2006, pp. 941-949. doi:10.1039/b514130c

[6] D. B. Shieh, C. H. Su, F. Y. Chang, Y. N. Wu, W. C. Su, J. R. Hwu, J. H. Chen and C. S. Yeh, "Aqueous Nickelnitrilotriacetate Modified $\mathrm{Fe}_{3} \mathrm{O}_{4}-\mathrm{NH}^{3+}$ Nanoparticles for Protein Purification and Cell Targeting," Nanotechnology, Vol. 17, No. 16, 2006, pp. 4174-4182. doi:10.1088/0957-4484/17/16/030

[7] C. C. You, A. Verma and V. M. Rotello, "Engineering the Nanoparticle-Biomacromolecule Interface," Soft Matter, Vol. 2, No. 3, 2006, pp. 190-204.

[8] N. E. Miller, "Associations of High-Density-Lipoprotein Subclasses and Apolipoproteins with Ischemic-Heart- Disease and Coronary Atherosclerosis," American Heart Journal, Vol. 113, No. 2, 1987, pp. 589-597. doi:10.1016/0002-8703(87)90638-7

[9] W. P. Castelli, R. D. Abbott and P. M. McNamara, "Summary Estimates of Cholesterol Used to Predict Coronary Heart-Disease," Circulation, Vol. 67, No. 4, 1983, pp. 730-734. doi:10.1161/01.CIR.67.4.730

[10] G. G. Rhoads, C. L. Gulbrandsen and A. Kagan, "SerumLipoproteins and Coronary Heart-Disease in a Population Study of Hawaii Japanese Men," New England Journal of
Medicine, Vol. 294, No. 6, 1976, pp. 293-298. doi:10.1056/NEJM197602052940601

[11] C. Edelstein, D. Pfaffinger and A. M. Scanu, "Advantages and Limitations of Density Gradient Ultracentrifugation in the Fractionation of Human-Serum Lipoproteins-Role of Salts and Sucrose," Journal of Lipid Research, Vol. 25, No. 6, 1984, pp. 630-637.

[12] R. J. Havel, H. A. Eder and J. H. Bragdon, "The Distribution and Chemical Composition of Ultracentrifugally Separated Lipoproteins in Human Serum," The Journal of Clinical Investigation, Vol. 34, No. 9, 1955, pp. 13451353. doi:10.1172/JCI103182

[13] D. W. Swinkels, H. L. M. Haklemmers and P. N. M. Demacker, "Single Spin-Density Gradient Ultracentrifugation Method for the Detection and Isolation of Light and Heavy Low-Density-Lipoprotein Subfractions," Journal of Lipid Research, Vol. 28, No. 10, 1987, pp. 1233-1239.

[14] T. Tadey and W. C. Purdy, "Chromatographic Techniques for the Isolation and Purification of Lipoproteins," Journal of Chromatography B: Biomedical Sciences and Applications, Vol. 671, No. 1-2, 1995, pp. 237-253. doi:10.1016/0378-4347(95)00051-J

[15] K. H. Weisgraber, T. P. Bersot, R. W. Mahley, G. Franceschini and C. R. Sirtori, "A-I Milano Apoprotein Isolation and Characterization of a Cysteine-Containing Variant of the A-I Apoprotein from Human High Density Lipoproteins," The Journal of Clinical Investigation, Vol. 66, No. 5, 1980, pp. 901-907. doi:10.1172/JCI109957

[16] X. Wu, R. H. Pelton, A. E. Hamielec, D. R. Woods and W. McPhee, "The Kinetics of Poly(N-Isopropylacrylamide) Microgel Latex Formation," Colloid and Polymer Science, Vol. 272, No. 4, 1994, pp. 467-477. doi:10.1007/BF00659460

[17] I. Lynch, I. Miller, W. M. Gallagher and K. A. Dawson, "Novel Method to Prepare Morphologically Rich Polymeric Surfaces for Biomedical Applications via Phase Separation and Arrest of Microgel Particles," Journal of Physical Chemistry B, Vol. 110, No. 30, 2006, pp. 1458114589. doi:10.1021/jp061166a

[18] C. Oslakovic, M. J. Krisinger, A. Andersson, M. Jauhiainen, C. Ehnholm and B. Dahlback, "Anionic Phospholipids Lose Their Procoagulant Properties When Incorporated into High Density Lipoproteins," Journal of Biological Chemistry, Vol. 284, No. 9, 2009, pp. 5896-5904. doi:10.1074/jbc.M807286200

[19] T. Berggård, et al., "140 Mouse Brain Proteins Identified by $\mathrm{Ca}^{2+}$-Calmodulin Affinity Chromatography and Tandem Mass Spectrometry," Journal of Proteome Research, Vol. 5, No. 3, 2006, pp. 669-687.

[20] T. Cedervall, I. Lynch, M. Foy, T. Berggård, S. C. Donnelly, G. Cagney, S. Linse and K. A. Dawson, "Detailed Identification of Plasma Proteins Adsorbed on Copolymer Nanoparticles," Angewandte Chemie International Edition, Vol. 46, No. 30, 2007, pp. 5754-5756. doi:10.1002/anie.200700465

[21] T. Cedervall, I. Lynch, S. Lindman, T. Berggard, E. Thulin, H. Nilsson, K. A. Dawson and S. Linse, "Understand- 
ing the Nanoparticle-Protein Corona Using Methods to Quantify Exchange Rates and Affinities of Proteins for Nanoparticles," Proceedings of the National Academy of Sciences of the United States of America, Vol. 104, No. 7, 2007, pp. 2050-2055. doi:10.1073/pnas.0608582104
[22] M. Lundqvist, I. Sethson and B. H. Jonsson, "Protein Adsorption onto Silica Nanoparticles: Conformational Changes Depend on the Particles' Curvature and Protein Stability," Langmuir, Vol. 277, No. 20, 2004, pp. 10639 10647.

\section{ABBREVIATIONS}

ApoA-I: Apolipoprotein A-I; RCF: Relative Centrifugal Force; PC: Phosphatidyl Choline. 